\title{
Excitation that lasts
}

The enticing playground of van der Waals structures offers new ways to engineer and manipulate excitons.

$\mathrm{T}$ he atomic-scale Legoland of twodimensional (2D) materials has become a much-frequented scientific playground, with building blocks aplenty to create and explore virtually unlimited stacking configurations. When two or more individual monolayers are placed together to form an artificial homo- or heterostructure with weakly interacting constituent layers, that is where the real van der Waals (vdW) architecture begins. Such creations offer electronic, optical and magnetic phenomena that are often utterly unlike the monolayer case. One notable example is the emergence of a new type of exciton in which the electron and the hole are spatially located in different layers. While the formation of these 'interlayer' or 'indirect' excitons is not exclusive to vdW structures, such as transition metal dichalcogenide (TMD) bilayers in which these species have been observed so far, the unique properties of the constituent $2 \mathrm{D}$ materials stemming from the intrinsic valley physics and the enhanced Coulomb interactions at the 2D limit make for an exciting research journey. Likewise, the much-discussed idea of hightemperature exciton condensation using long-lived interlayer excitons remains a possibility that is yet to be experimentally explored. In contrast to monolayers, vdW layered structures possess an additional degree of freedom: the twist angle that stems from the sensitive dependence of the exciton properties on the interlayer interaction and the structural alignment of the constituent monolayers. This unique feature is of particular importance from the perspective of practical applications as it offers a new means of engineering and manipulation of interlayer excitons.

In this issue, Nature Nanotechnology takes the readers on a tour of the many fundamental and applied aspects of interlayer exciton phenomena in vdW homoand heterostructures, presenting those interested in the subject with the most recent advances in the sub-field and its envisaged technological prospects. In our first Review article, the pioneers of interlayer exciton research, Xiaodong Xu and colleagues, provide a thorough examination of exciton phenomena in TMD heterobilayers. Starting from the contemporary theoretical description of interlayer excitons, they recount the available experimental observations that began with the detection of optically active valley interlayer excitons in a TMD type-II heterostructure and progressed to the current understanding of exciton formation, dynamics and manipulation.

Whereas on long timescales the optical properties and exciton dynamics in $\mathrm{vdW}$ heterostructures are dominated by recombination of interlayer excitons and relaxation of the spin and valley degrees of freedom, charge transfer in TMD vertical stacks is a much faster process that has been shown to mediate the formation of interlayer excitons, making such ultrafast phenomena of critical importance in the frame of interlayer exciton dynamics. Following a natural evolution of studies on monolayer 2D materials towards more complex vdW heterostructures, Tony Heinz and Feng Wang summarize in their Review the recent progress in excited-state dynamics in TMD heterostructures, such as two competing ultrafast processes (charge transfer and energy transfer between the TMD layers) as well as the spin and valley dynamics that are instrumental for the realization of future spintronic and valleytronic devices. Once again, the authors point out that the choice of the constituent monolayers, their relative alignment and the quality of the heterostructures strongly influence the ultrafast dynamics that directly affects the properties of interlayer excitons. One of the notable traits of interlayer excitons, their extended lifetime, offers the possibility of electrostatic exciton manipulation, a direction that has been relatively well explored in relation to interlayer exciton control. In their Commentary, Kin Fai Mak and Jie Shan discuss the quantum-confined
Stark effect in TMD bilayers and look at its unique features which set $\mathrm{vdW}$ heterostructures apart from other coupled quantum wells that support interlayer exciton formation. Simultaneously, the authors ponder new ways of interlayer exciton control by means such as structural tuning or applied pressure, which, similar to a relative twist angle, is expected to alter optoelectronic properties and moiré mini-bands.

From an even broader perspective, the prodigious progress in vdW homo- and heterostructures calls for a systematic examination of available and emerging design strategies for engineering the properties of this new class of synthetic quantum materials. Justin Song and Nathaniel Gabor go beyond exciton phenomena by looking at vdW structures as electron quantum metamaterials and take on the challenge by providing a summary of the tools for tuning quantum behaviour in vdW structures. Such tuning could be done by preparing the systems with tailored unitcell size and wavefunction texturing, or by manipulating interlayer coupling and using Coulomb fields engineering.

With so many possibilities in store, the bright future of vdW heterostructure physics almost sounds like a self-fulfilling prophecy. Quite possibly, the same goes for the interlayer exciton prospects. Their temporal stability even at high temperatures, their valley pseudospin and the moiré superlattice effects could lead to the realization of a degenerate Bose gas and excitonic superfluidity, rendering interlayer excitons potentially practical for power-efficient optoelectronic and valleytronic devices. In the meantime, we leave our readers to appreciate the valuable efforts that have been made so far towards a better fundamental understanding of these peculiar long-lasting excitations.

Published online: 5 November 2018 https://doi.org/10.1038/s41565-018-0316-7 